\title{
Academic Burnout and Motivation of Chinese Secondary Students
}

\author{
Xiaozhou Zhang, Robert M. Klassen, and Yun Wang
}

\begin{abstract}
The purpose of this study was to identify patterns of academic burnout in 730 Chinese middle school students, and to link the burnout patterns with academic motivation. Using cluster analysis, we identified four motivation groups: (a) distressed group, (b) persevering group, (c) laissez-faire group, and (d) well-functioning group. The results of optimal scaling procedures indicated that grade level and motivation variables (intrinsic and extrinsic motivation, and amotivation) were good discriminators among the four clusters. Specifically, variation on self-determination motivation characteristics was demonstrated among different academic burnout profiles: distressed students had the highest scores on a motivation and external motivation. In contrast, well-functioning students had the highest scores on intrinsic motivation. For persevering and laissez-faire students, there was no significant difference on intrinsic motivation. However, persevering students reported higher scores on a motivation and external motivation than laissez-faire students.
\end{abstract}

Index Terms-Burnout, motivation, profile, Chinese students.

\section{INTRODUCTION}

Maslach (1981) defined burnout as a syndrome of emotional exhaustion and cynicism that occurs among individuals who performed "people work" of some kind (e.g., health care, education, and social work) [1]. Lately, the concept of burnout was expanded to include all types of students. Students' academic burnout exists because students' core activities can be considered as a kind of "work" [2]-[5]. Academic burnout is hypothesized to consist of three conceptually distinct but empirically related dimensions: exhaustion due to study demands, cynical and detached attitude toward one's studies, and feelings of incompetence as a student [3], [6].

The multidimensional theory of burnout is presumed to include the dimension of exhaustion plus two other dimensions such as response toward others (cynicism), and response toward self (reduced personal accomplishment) [7]. In addition, there are two more major theoretical approaches informed the study of profiles of academic burnout: the Job Demands-Resources model (JD-R) [8] and process models [9]. Based on these theories, it is reasonable to propose that burnout profiles consisting of multiple clusters should be

Manuscript received November 15, 2012; revised January 16, 2013.

Xiaozhou Zhang is with the Department of Educational Psychology, University of Alberta, Canada, T6G1K7 (e-mail: xzhang15@ualberta.ca).

Robert Klassen is with the Psychology in Education Research Center, University of York, YO10 5DD (e-mail: robert.klassen@york.ac.uk).

Yun Wang is with the Institution of Cognitive Neuroscience and learning, Beijing Normal University, China, 100875 (e-mail: wangyun@bnu.edu.cn). explored rather than the uni-dimensional clusters. Person-oriented approaches have been used in multicultural educational setting to explore academic motivation in Canada [10], academic coping in the United States [11], achievement goal orientation in Finland [12] and academic burnout in South Korea [13]. Across these studies, focusing on individuals instead of variables allowed for the identification of homogeneous groups of students who share similar psychological characteristics. It is particularly important that research investigating burnout include the exploration of multiple dimensions.

Many Chinese parents agonize over their children's motivational profile-“'I'm worried that my child is not motivated at school" - but according to Self-Determination Theory (SDT), motivation beliefs are not global and undifferentiated, and it is the quality of motivation that is the key to academic success [14]. SDT conceptualizes motivation from amotivation or unmotivated to extrinsic motivation to intrinsic motivation that reflects the extent to which behavior originates from the individual in question.

Deci \& Ryan [15] addressed that social contexts people embedded in will affect their varied basic needs and type of motivation, which thus influence their wellness and effectiveness. A number of empirical studies have focused on examining the relationship between motivation and mental health issues such as stress [16], [17], subjective well-being [12], [18], and burnout [19], [20]. Little research focused on relationship between academic burnout and motivation on a person-oriented perspective. The purpose of the current study is to identify profiles of academic burnout among Chinese adolescents and also to explore the gender, grade and motivation differences among various types of academic burnout.

\section{METHODS}

\section{A. Participants and Procedure}

The participants in this study were 730 adolescents (44\% female) enrolled in one middle school and one regular secondary school in northern China. The schools were chosen according to convenient and random policy. The schools were in a middle SES area of the city, and almost all students at the schools were from the local community.

In China, the education is divided into three categories: basic education including pre-school, primary and regular secondary education; higher education; adult education. In this study, middle school participants were 130 seventh grade students (43\% female), 131 eighth grade students $(40 \%$ female), and 146 ninth grade students (45\% female). 
Secondary school students were 123 tenth grade students (43\% female), 124 eleventh grade students (49\% female), and 76 twelfth grade students (43\% female). Participants were volunteers who completed a self-report questionnaire on motivation and burnout during a break between classes. Response rate was $98.6 \%$ and the questionnaires were administered in the classroom and collected immediately upon completion.

\section{B. Measurements}

\section{1) Academic motivation}

We used a 30-item version of the Academic Self-Regulation Questionnaire (SRQ-A), previously used in Asian contexts by Lee, et al. (2012) in Korea [21]. The scale was created by Ryan and Connell (1989) in the United States [22]. The SRQ-A consists of five scales: external regulation (6 items); introjected regulation (6 items); identified regulation (6 items); intrinsic motivation (6 items); amotivation (6 items). All items were scored on a 6-point Likert scale from "very untrue" to "very true". Previous versions of the SRQ-A were showed good psychometric properties, with evidence of reliability (Cronbach's alpha range from .76 to .81) and validity [22]. The questionnaire was translated into Chinese by the first author, with the validity of the translation ensured by careful back-translation conducted by two bilingual experts.

In the present study, internal consistency for the scores of all 30 items was .78 and the reliability coefficients for each of the subscale scores were: 0.83 for external regulation, 0.77 for introjected regulation, 0.85 for identified regulation, 0.91 for intrinsic motivation, and 0.84 for amotivation. Confirmatory factor analysis (CFA) supported the construct validity of the translated measure $\left(\chi^{2} / \mathrm{df}=2.96, C F I=.93\right.$, RMSEA=.05).

\section{2) Academic burnout}

The Maslach Burnout Inventory-Student Survey (MBI-SS) [6] is a 15-item version of Maslach Burnout Inventory (MBI) adapted for use with students. Hu \& Schaufeli (2009) examined the factorial validity of the Chinese version of the MBI-SS with results indicating that the scale could be used to assess burnout in Chinese students [23]. The MBI-SS includes three scales: exhaustion (EX; 5 items), cynicism (CY; 4 items), and efficacy (EF; 6 items). All items re scored on a 7-point Likert scale from 0 (never) to 6 (always). Higher scores on EX and CY and low scores on EF indicate higher levels of burnout. Values of Cronbach alpha in the Chinese sample were acceptable [23]. The questionnaire was adapted according to middle schooling cultural background in this study.

In the present study, internal consistency for the scores of all 15 items was .88 (Cronbach's alpha), with reliability coefficients for the subscale scores as follows: .81 for exhaustion, .84 for cynicism, and .84 for efficacy. Confirmatory factor analysis (CFA) supported the construct validity of the translated measure $\left(\chi^{2} / \mathrm{df}=3.84, C F I=.95\right.$, RMSEA=.06).

\section{Data Analysis}

For the academic burnout profiles, students with similar patterns of academic burnout were identified through K-Means Cluster Analysis. In this analytic approach, K-Means Cluster Analysis is a method of cluster analysis which aims to partition $\mathrm{n}$ observations into $\mathrm{k}$ clusters in which each observation belongs to the cluster with the nearest mean. After having established the different academic burnout groups, Optimal Scaling Procedure was used to discover which variables contributed to cluster group separation. The idea behind Optimal Scaling Procedure is to assign numerical quantifications to the categories of each variable, thus allowing standard procedures to be used to obtain a solution on the quantified variables. Academic burnout profiles, gender and grade were defined as nominal and the dimensions of motivation (external, introjected, identified, intrinsic, amotivation) were defined as numeric in the analysis. Finally, ANOVA and Chi-square analyses were conducted in order to examine burnout group differences in terms of motivation, gender, and grade.

\section{RESULTS}

\section{A. Academic Burnout Profiles}

Before analyzing the data, missing values were replaced using linear interpolation and the items of the efficacy scale on the MBI-SS were reversed (and renamed "Efficacy Reduction") in order to ease comparison with the other MBI-SS scales. The three subscales of MBI-SS were standardized, and then K-Means Cluster Analysis was used to replicate the hypothesized four groups of Academic Burnout (i.e., distressed, laissez-faire, persevering, and well-functioning) as proposed by Lee et al., (2010). The results of the cluster analysis of Academic Burnout are presented in Fig. 1.

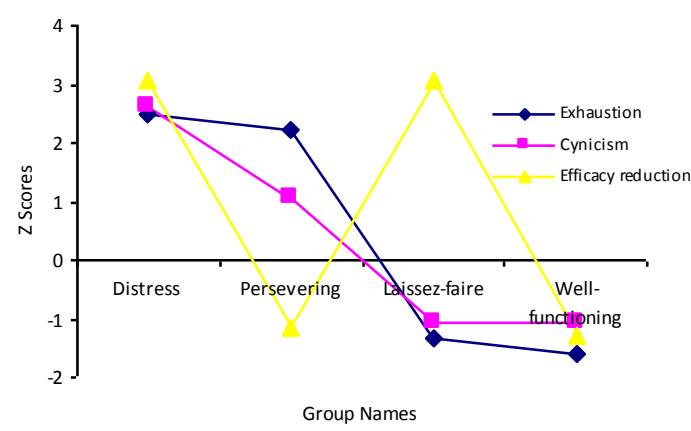

Fig. 1. Empirically derived academic burnout clusters as a function of the academic burnout measurement $Z$ scores.

Results showed that the first cluster-distressed students- $(n=92,12.6 \%$ of students $)$ had high exhaustion, cynicism and efficacy reduction, suggesting overall high levels of burnout, coupled with little confidence in their capabilities to ameliorate the situation.

The second cluster of students - persevering students - $(n$ $=177,24.2 \%$ of students) had high levels of exhaustion and cynicism, coupled with low efficacy reduction, suggesting a group of students who report high levels of stress (i.e., exhaustion and cynicism), but who retain confidence in their capabilities.

The third cluster-laissez-faire students- $(n=132,18.1 \%$ 
of students) displayed high efficacy reduction, coupled with low scores on exhaustion and cynicism. The profile of this cluster of students suggests a group with low confidence, which may put less effort and focus on their study in order to avoid feeling distress. Graphically, the shape of this cluster presents a mirror image of students in the persevering group.

The forth cluster-well-functioning students- $(n=329$, $45.1 \%$ of students) reported low exhaustion, cynicism and efficacy reduction scores. The profile of this cluster of students suggests a group that presents with high engagement for studying, and the belief that studying will increase their academic achievement. Graphically, the shape of this cluster provides a mirror image of students in the distressed group.

\section{B. Contribution of Motivation to Separation of Burnout Clusters}

We used optimal scaling procedure to investigate the variables that contributed to separation of the four burnout clusters. To that end, we examined how gender, grade, and six types of motivation (intrinsic, integrated, identified, introjected, external, and amotivation) contributed to cluster group separation. The four clusters of academic burnout, gender, and grade level were defined as nominal level, and self-determination motivation was defined at the numeric level. The main results of the optimal scaling regression are presented in Table I.

\begin{tabular}{|c|c|c|c|c|c|c|}
\hline \multirow[t]{2}{*}{ Variables } & \multicolumn{2}{|c|}{$\begin{array}{l}\text { Standardized } \\
\text { Coefficients }\end{array}$} & \multicolumn{2}{|c|}{ Correlations } & \multirow[t]{2}{*}{$\mathrm{F}$} & \multirow[t]{2}{*}{ Tol } \\
\hline & Beta & $\begin{array}{l}\text { Std. } \\
\text { Error }\end{array}$ & Zero & $\mathrm{Pa}$ & & \\
\hline Gender & .02 & .03 & .09 & .02 & .31 & .97 \\
\hline Grade & .09 & .03 & .07 & .12 & $10.84 * * *$ & .95 \\
\hline External & -.19 & .04 & -.46 & -.20 & $29.03 * * *$ & .63 \\
\hline Introjected & -.01 & .03 & -.12 & -.01 & .03 & .80 \\
\hline Identified & .02 & .04 & .35 & .02 & .39 & .51 \\
\hline Intrinsic & .17 & .04 & .46 & .16 & $19.24 * * *$ & .50 \\
\hline Amotivation & -.42 & .04 & -.61 & -.41 & $148.93 * * *$ & .65 \\
\hline
\end{tabular}

\begin{tabular}{l}
\hline \hline Note1: $* * * P<0.001 ;$ Pa means Partial; Tol means Tolerance \\
Note2: Total $R^{2}=0.44$; Adjusted $R^{2}=0.43 ; F_{(11,718)}=51.26 ; P<0.001$
\end{tabular}

The regression model accounted for $43.1 \%$ of the variance of cluster group with five variables (gender, grade and three sub-dimensions of self-determination motivation). Amotivation had the largest absolute coefficient (Beta $=-.423$, $p<0.001)$, followed by external (Beta=-.189, $p<0.001)$ and intrinsic $($ Beta $=.174, p<0.001)$. Grade was also statistically significant.

In Table I, amotivation had the partial correlation of -0.414 that indicates it could explain $17.140 \%$ of the variance of the clusters controlling effects of other variables. External and intrinsic had the partial correlation of -0.197 and 0.162 that means they could account for $3.881 \%$ and $2.624 \%$ of the variance of the clusters removing effects of other variables. Grade could explain $1.488 \%(0.122)$ as well.

For the column of tolerance which examines multicollinearity among predictors, all of the values were larger than 0.45 which was larger than the critical value 0.2 that meant that all of the predictors were not correlated to each other very well and the problem of multicollinearity could be ignored.

Then $\chi^{2}$ and ANOVA analyses were calculated to figure out the characteristics of Academic Burnout in terms amotivation, external and intrinsic motivation. The results were demonstrated in Table II.

TABLE II: CLUSTER CHARACTERISTICS OF ACADEMIC BURNOUT AMONG AMOTIVATION, EXTERNAL, INTRINSIC

\begin{tabular}{|c|c|c|c|}
\hline & AMOTIVATION & EXTERNAL & INTINSIC \\
\hline $\mathrm{DS}^{1}$ & $3.88 / 1.19$ & $2.81 / 1.17$ & $2.85 / 1.18$ \\
\hline $\mathrm{PS}^{2}$ & $2.44 / 0.99$ & $2.19 / 1.01$ & $3.98 / 1.05$ \\
\hline $\mathrm{LFS}^{3}$ & $1.98 / 0.85$ & $1.76 / 0.78$ & $3.73 / 1.28$ \\
\hline $\mathrm{WFS}^{4}$ & $1.51 / 0.66$ & $1.44 / 0.61$ & $4.78 / 0.98$ \\
\hline $\mathrm{F}$ & $166.23 * * *$ & $69.85 * * *$ & $79.76^{* * *}$ \\
\hline$\eta 2$ & 0.41 & 0.22 & 0.25 \\
\hline Contrasts & $1>2,3,4$ & $2>3,4 ; 3>4$ & $\begin{array}{l}4>1,2,3 ; \\
3>1 ; 2>1\end{array}$ \\
\hline
\end{tabular}

Note: $* * * P<0.001$

It revealed that there were significant differences of grade on cluster classification $\left(\chi^{2}=48.887, P<0.001\right)$. Specifically, the percentage of 'Well-functioning students' decreased from $66.9 \%$ to $34.2 \%$ and 'Distress students' increased from $3.8 \%$ to $17.1 \%$ along with the increasing of grade level. Comparing to other grade levels, eighth students took up the highest percentage (26\%) of the laissez-faire group who had lower scores on exhaustion and cynicism and higher scores on efficacy reduction; the tenth students took up the highest percentage $(35 \%)$ of the persevering group who had higher scores on exhaustion, cynicism and lower scores on efficacy reduction.

It showed that there were significant differences of clusters on amotivation $(F=166.230)$, External $(F=69.848)$ and Intrinsic $(F=79.756)$. Specifically, distressed students had the highest scores on amotivatoin and external, in contrast, Well-functioning students had the highest scores on Intrinsic. For persevering and Laissez-faire students, there were no significant differences on Intrinsic. However, Persevering students reported higher scores on amotivation and external than the laissez-faire students.

\section{DISCUSSION}

Based on the response of Chinese students, participants were classified into four identified groups: Distressed, Persevering, Laissez-faire and Well-functioning groups that supported Hypothesis 1, consistent with the previous research based on Korean sample.

The highest percentage of cluster was the well-functioning group $(45.1 \%)$ which was an optimistic result. Students in this group reported lowest scores on exhaustion, cynicism and efficacy reduction. Most of them had extra energy and they would like to put efforts on academic study because of their sufficient confidence on competence about studying. However, there were also $12.6 \%$ students who were classified into the distressed group in which students reported the highest scores on exhaustion, cynicism and efficacy reduction. Students in this group may suffer from emotional exhaustion, lack of enthusiasm on academic study and 
demonstrate helplessness regarding their studies to some extent.

Moreover, there were also $24.2 \%$ and $18.1 \%$ students who were classified into 'persevering students' who felt distress but they had confidence and were still hard working on their study and 'laissez-faire students' who had less confidence and may put less effort and focus on their studying in order to avoid to feeling distress, respectively. For persevering and Laissez-faire students, there are no significant differences on intrinsic motivation. It means different results would be led to by the same level of intrinsic motivation. However, Persevering students reported higher scores on amotivation and external motivation than Laissez-faire students that indicates students who have no motivation or motivated by the outside would have an influence on individuals' emotional experience, not self-efficacy. More variables should be involved to predict the burnout profiles clearly.

\section{CONCLUSION}

Four types of students were identified concerning academic burnout: distress, persevering, laissez-faire and well-functioning. In addition, distressed students had the highest scores on amotivatoin and external motivation; well-functioning students had the highest scores on intrinsic motivation. No significance with intrinsic motivation between persevering and laissez-faire students. However, Persevering students reported higher scores on amotivation and external than laissez-faire students.

Although some interesting results were revealed, some limitations could be improved in the future. 1) Longitudinal research should be conducted in future to examine causeeffect relationship; 2) academic performance should be involved to solve practical problems in school setting; 3) cross-cultural research could be considered and there would be different profile types by demographics and other variables.

\section{IMPLICATION}

This study contributes to current research on academic burnout in three main respects.

First, the study is among the first to examine burnout and motivation in Chinese secondary school students.

Second, the results of the person-centered analysis replicate the four distinct groups of students earlier proposed by Lee (2010) [13], and shows distinct motivation profiles for these four groups.

Third, results revealed that the percentage of distressed students increased with grade level through middle and high school. The result was consistent with previous research [24] [25] which found that shows motivation declining for some students through adolescence. Conservation of Resources theory (COR) [26], [27] suggests that individuals will experience burnout when requirements and resources became unbalanced, especially when there are increasing requirements but declining support. In addition, based on the Stage-Environment Fit theory, Grimshaw, et al., [28] argued that the school's environment needs to include additional support as requirements change and students' interests and engagement change as they mature. If these changes are not made, students will disengage psychologically and then physically from school. As adolescents progress through school, additional demands from academic tasks are coupled with no change in level of psychological support, leading to our finding of a higher percentage of distressed students in higher grades.

\section{REFERENCES}

[1] C. Maslach and S. E. Jackson, "The Measurement of Experienced Burnout," Journal of occupational behavior, vol. 2, pp. 99-113, April 1981.

[2] N. Kiuru, K. Aunola, E. Leskinen, and K. S. Aro, "Peer Group Influence and Selection in Adolescents' School Burnout: A Longitudinal Study," Merril-Palmer Quarterly, vol. 54, pp. 23-55, Jan 2008 .

[3] K. S. Aro, N. Kiuru, E. Leskinen, and J. Nurmi, "School Burnout Inventory (SBI): Reliability and Validity," European Journal of Psychological Assessment, vol. 25, pp. 48-57, 2009.

[4] S. S. Shih, "An examination of academic burnout versus work engagement among Taiwaness adolescents," The Journal of Educational Research, vol. 105, pp. 286-298, June 2012.

[5] C. S. Galbraith and G. B. Merrill, "Academic and work-related burnout: a longitudinal study of working undergraduate university business students," Journal of College Student Development, vol. 53, pp 453-463, May-June 2012.

[6] W. B. Schaufeli, I. M. Martinez, A. M. Pinto, M. Salanova, and A. B. Bakker, "Burnout and Engagement in University Students: A Cross-National Study," Journal of Cross-Cultural Psychology, vol. 33 pp. 464-481, Sep 2002.

[7] C. Maslach, "A multidimensional theory of burnout," in Theories of Organizational Stress, Cooper, C. L. Ed., Oxford University Press, Oxford, pp. 68-85, 1998.

[8] A. B.Bakker and E. Demerouti, "The job demands-resources model: state of the art," Journal of Managerial Psychology, vol. 22, pp. 309-28, 2007.

[9] R. T. Lee and B. E. Ashforth, "A meta-analytic examination of the correlates of the three dimensions of job burnout," Journal of Applied Psychology, vol. 81, pp. 123-133, Apr 1996.

[10] C. F. Ratelle, F. Guay, R. J. Vallerand, S. Larose, and C. Senécal "Autonomous, controlled, and amotivated types of academic motivation: A person-oriented analysis," Journal of Educational Psychology, vol. 99, pp. 734-746, Nov 2007.

[11] A. A. Aldridge and S. C. Roesch, "Developing coping typologies of minority adolescents: A latent profile analysis," Journal of Adolescence, vol. 31, pp. 499-517, August 2008.

[12] H. T. Soini, K. S. Aro, and M. Niemivirta, "Achievement goal orientations and subjective well-being: A person-centred analysis," Learning and Instruction, vol. 18, pp. 251-266, June 2008.

[13] J. Lee, A. Puig, Y. Kim, H. Shin, J. H. Lee, and S. M. Lee, "Academic Burnout Profiles in Korean Adolescents," Stress and Health, vol. 26, pp. 404-416, Dec 2010.

[14] F. Guay, C. F. Ratelle, and J. Chanal, "Optimal learning in optimal contexts: the role of self-determination in education," Canadian Psychology, vol. 49, pp. 233-240, Aug 2008.

[15] E. L. Deci and R. M. Ryan, "Motivation, personality and development within embedded social contexts: an overview of self-determination theory," in The Oxford Handbook of Human Motivation, Ryan, R. M., New York: Oxford University Press, pp.85-107, 2012

[16] S. R. Baker, "Intrinsic, extrinsic, and amotivational orientations: Their role in university adjustment, stress, well-being, and subsequent academic performance," Current Psychology, vol. 23, pp. 189-202, 2004.

[17] J. Park, S. Chung, H. An, S. Park, C. Lee, S. Y. Kim, J. Lee, and K. Kim,"A structural model of stress, motivation, and academic performance in medical students," Psychiatry Investing, vol. 9, pp. 143-149, June 2012.

[18] K. M. Sheldon, "The self-determination theory perspective on positive mental health across cultures," World Psychiatry, vol. 11, pp. 101-102, June 2012.

[19] C. T. Pisarik, "Motivational orientation and burnout among undergraduate college students," College Student Journal, vol. 43, pp. 1238-1252, Dec 2009 
[20] S. I. Gautheur, E. G. Descas, and P. N. Lemyre, "A prospective study of the influence of perceived coaching style on burnout propensity in high level young athletes: using a self-determination theory perspective," Sport Psychologist, vol. 26, pp. 282-298, 2012.

[21] J. Lee, H. Yu, and S. Choi, "The influences of parental acceptance and parental control on school adjustment and academic achievement for South Korean children: the mediation role of self-regulation," Asia Pacific Education Review, vol. 13, pp. 227-237, June 2012.

[22] R. M. Ryan and J. P. Connell, "Perceived locus of causality and internalization: examining reasons for acting two domains," Journal of Personality and Social Psychology, vol. 57, pp. 749-761, Nov. 1989.

[23] Q. Hu and W. B. Schaufeli, "The factorial validity of the Maslach burnout inventory-student survey in China," Psychologist, vol. 105, pp. 394-408, Oct 2009.

[24] J. S. Eccles, C. Midgley, A. Wigfield, C. M. Buchanan, D. Reuman, C. Flanagan, and D. MacIver, "Development during adolescence: The impact of stage-environment fit on young adolescents' experiences in schools and in families," American Psychologist, vol. 48, pp. 90-101, Feb 1993.

[25] L. X. Yang, "Academic burnout and its relationship with academic commitment among college students," M.S. thesis, Dept. Education. Fujian Teaching Univ., Fujian, China, 2004.

[26] S. E. Hobfoll, "Conservation of resources: a new attempt at conceptualizing stress," American Psychologist, vol. 44, pp. 513-524, Mar 1989.

[27] S. E. Hobfoll, The psychology and philosophy of stress, culture and community, New York: Plenum Press, 1998, ch2.

[28] J. M. Grimshaw, R. E. Thomas, G. MacLennan, C. Fraser, C. R. Ramsay, L. Vale, P. Whitty, M. P. Eccles, L. Matowe, L. Shirran, M Wensing, R. Dikstra, C. Donaldson, and A. Hutchinson, "Effectiveness and Efficiency of Guideline Dissemination and Implementation Strategies," Health Technology Assessment Report, vol. 8, pp.1-72, 2004.

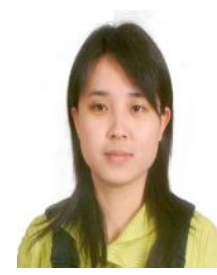

Xiaozhou Zhang was born in Changzhi, Shanxi Province, China, on Sep $24^{\text {th }}$, 1985. I graduated university and gained Bachelor degree of Science in 2006 in Beijing Normal University (BNU), Beijing, China. Then I was working on my Master thesis from 2006 to 2010 in BNU on academic burnout based on cultural perspective. From 2008 to 2009, I studied in Ewha Woman University, Seoul, South Korea as an exchange student. I completed data collection of Korean sample during this year. I am PhD student in Educational Psychology Department, University of Alberta, Canada. She worked in various Non-profit organizations as a volunteer such as Zigen Fund for Grassroots Development, Beijing (Mar. 2007-Oct. 2008), and Shine Youth Clinic, Edmonton (May.2011-present) She also did some internship work such as in Beijing Furen Applied Psychology Organization as a Counseling Assistant, Beijing (2006). She presented some papers in international conferences and published two journal papers in Chinese. Her research focuses on psychosocial features and emotion problems during the period of transitioning into adulthood from cultural and developmental perspectives.Ms. Zhang is a member of the American Psychological Association, the International Society for the Study of Behavioral Development, the Society for Research in Child Development, and the Canadian Psychological Association. 